\title{
INDIVIDUAL DIFFERENCES IN LOGICAL INTUITIONS ON REASONING PROBLEMS PRESENTED UNDER TWO-RESPONSE PARADIGM
}

\author{
Roman Buriča \& Jakub Srol ${ }^{b}$ \\ ${ }^{a}$ Department of Psychology, Faculty of Arts, Comenius University in Bratislava \\ ${ }^{\mathrm{b}}$ Institute of Experimental Psychology, Centre of Social and Psychological Sciences, Slovak \\ Academy of Sciences
}

This is an unpublished working paper (December 13, 2019).

Corresponding author:

Jakub Šrol

Institute of Experimental Psychology, Centre of Social and Psychological Sciences, Slovak Academy of Sciences

Dúbravská cesta 9, 84104 Bratislava, Slovakia

Phone: + 421 - 2 - 54775625

Email: jakub.srol@savba.sk

\section{ACKNOWLEDGMENTS}

The research presented here is based on an unpublished doctoral dissertation of the second author (J.Š.). The study was supported by the Slovak Research and Development Agency and is part of the research project APVV-16-0153: "Cognitive failures - individual predictors and intervention possibilities". The data for this study are publicly available at: https://osf.io/bvzhu/ 


\begin{abstract}
Studies on individual differences in susceptibility to cognitive biases have identified several cognitive dispositions which were thought to predict reasoning by contributing to the efficiency of analytic thought. Recently formulated hybrid models, however, suggest that substantial differences between reasoners may arise early already in the intuitive stages of the reasoning process. To address this possibility, we examined standard individual difference measures, mindware instantiation, and conflict detection efficiency as predictors of the accuracy on conflict reasoning problems presented under a two-response paradigm. This was intended to tease apart the predictors of intuitive responding from those factors which only contribute to reasoning when participants have enough time for analytic engagement. We found that participants correctly solved almost half of conflict reasoning problems already at the initial response stage and that the individual differences in initial reasoning performance were predicted by their cognitive reflection, mindware instantiation, and detection efficiency. The findings advance the specification of hybrid dual-process models and provide corroborating evidence that a part of the link between bias susceptibility and cognitive dispositions is due to differences in intuitive processing.
\end{abstract}

Keywords: Two-response paradigm. Hybrid model. Conflict detection. Mindware instantiation. Individual differences. 


\section{INDIVIDUAL DIFFERENCES IN LOGICAL INTUITIONS ON REASONING PROBLEMS PRESENTED UNDER TWO-RESPONSE PARADIGM}

\section{INTRODUCTION}

Since early studies by Tversky and Kahneman (1974), the research on heuristics and biases has shown us that people are capable of making remarkably sound inferences and decisions when facing situations in which they can make use of intuitions (Kahneman, 2011). Simultaneously, we have also learned that humans can be exceptionally inaccurate when they encounter problems which necessitate the use of logico-mathematical principles that do not align with their initial intuitive judgments. To account for the vast literature that has amassed in the research on cognitive biases, numerous dual-process models have been proposed which distinguished between two qualitatively different types of thought. Arguably the most dominant among such accounts was the so-called default-interventionist model under which reasoning and decision making were thought to reflect a sequential engagement of intuitive "type 1" and analytic "type 2" cognitive processes (Evans \& Stanovich, 2013; Kahneman, 2011; Stanovich, West, \& Toplak, 2011). At the early stages of reasoning, type 1 processes were thought to operate autonomously and without any conscious control or demands on executive resources produce an initial intuitive response often based on heuristics or simplified reasoning rules. Intuitive processing was thought to often provide reasonably accurate judgments and decisions without the need to engage in more demanding analytic thought, thus substantially saving our cognitive resources (Kahneman, 2011).

However, in situations which contain misleading intuitive cues, such as when reasoning with conflict problems traditionally used in the research on cognitive biases, initially produced intuitive response is likely to be biased and needs to be corrected by engaging in type 2 processing which is consciously controlled and thus loads heavily on one's working memory resources (Evans \& Stanovich, 2013). Under such circumstances, the default-interventionist model predicted that people first need to detect that their heuristic intuition is not in line with logical considerations of the task at hand. After successful conflict detection, reasoners could engage in more demanding analytic operations, such as inhibiting the intuitive response and deriving a normatively correct answer using formal logical knowledge - mindware - 
necessary to solve the particular problem (Evans \& Stanovich, 2013; Stanovich et al., 2011). Of course, one could engage in analytic thought only to rationalize the initial response or carry out shallow computations, such as serial associative cognition, in which case the final response after analytic engagement may not be any more normatively appropriate that the initial heuristic one (Pennycook, Fugelsang, \& Koehler, 2015; Stanovich, 2009).

An important line of research which informed the formulation and development of the default-interventionist model were the studies on individual differences in susceptibility to cognitive biases (e.g., Stanovich \& West, 2000; Stanovich, West, \& Toplak, 2016). Today, they provide abundant evidence that sound reasoning in various heuristics and biases tasks depends on factors such as cognitive ability, thinking dispositions, numeracy, and/or cognitive reflection (Klaczynski, 2014; Šrol \& De Neys, 2019; Toplak, West, \& Stanovich, 2011). Although it was recognized under the default-interventionist model that intuitive responses need not be biased and that the response brought with the use of type 2 processing will not necessarily be normatively correct (Evans, 2012), the role of abovementioned factors in sound reasoning performance was attributed solely to the engagement and/or efficiency of analytic thought. This was because the model assumed that few continuous differences between reasoners exist in the nature of intuitive processing (e.g. Evans \& Stanovich, 2013). Recently, however, evidence starts to emerge against this view (Thompson, Pennycook, Trippas, \& Evans, 2018) which suggests that substantial individual differences exist already at early stages of the reasoning process when analytic thought presumably could not yet have taken place. Here, we first review three lines of evidence which support the view that there are important differences between reasoners in intuitive processing. Then, we sketch our own attempt at examining individual difference factors which might contribute to the efficiency of intuitive logical thought and comparing them to those which only aid reasoning when people have enough time for engaging in more demanding analytic operations.

One of the core assumptions of the default-interventionist model was that people are mostly biased because they fail to detect that their heuristic intuition is not in line with the logical considerations of the task at hand as these considerations presumably come into play only later in the reasoning process after the engagement of analytic thought (De Neys, 2018). However, there is now a whole line of research which shows that even if people are eventually biased, they do indeed process problems which involve a conflict between heuristic intuition and logico-mathematical principles differently from those which do not contain such a conflict. Specifically, conflict detection studies show that in comparison with no-conflict 
tasks, on conflict problems people exhibit prolonged response times, lower confidence, lower feelings of rightness, and other psychological and neurophysiological changes which are indicative of their conflict sensitivity (for reviews, see De Neys, 2012, 2018). Moreover, this conflict detection ability seems to be largely unaffected by manipulations which limit the possibility for analytic engagement, such as instructions to respond intuitively, restricted time limit, or concurrent working memory load (Bago \& De Neys, 2017; Franssens \& De Neys, 2009; Johnson, Tubau, \& De Neys, 2016). Based on such evidence, conflict monitoring is now widely regarded to take place largely intuitively (De Neys, 2012, 2018; Pennycook, 2018; Pennycook et al., 2015). Importantly for the present study, in a recent research by Šrol and De Neys (2019), it was shown that the efficiency of conflict detection is related to several individual difference factors such as mindware instantiation (i.e. the automatization of logical knowledge necessary to solve the heuristics and biases tasks), cognitive reflection, and analytic thinking disposition (for partial findings, see also Frey, Johnson, \& De Neys, 2018; Pennycook, Cheyne, Barr, Koehler, \& Fugelsang, 2014; Pennycook et al., 2015). As the detection of conflict necessitates that one intuitively processes the rudimentary logical structure of the reasoning problem in order to realize that their heuristic intuition is not aligned with it (De Neys, 2018), the abovementioned studies could be taken as a first piece of evidence that some people are remarkably efficient in this form of intuitive logical processing.

Another piece of evidence against the default-interventionist account of individual differences in bias susceptibility comes from studies which employed a Stroop-like interference paradigm to study the reasoning process (Handley, Newstead, \& Trippas, 2011; Trippas, Thompson, \& Handley, 2017). In this line of research, participants are asked to judge various conflict and no-conflict reasoning problems either on the basis of their logical validity or believability. Contrary to the assumption that the judgment of logical validity in conflict tasks necessitates analytic engagement which presumably comes only after intuitive processing stage (Thompson \& Newman, 2018), logical validity of the inference was shown to interfere with belief-based judgments even more than believability of the inference did with the validity judgments (Handley et al., 2011). Even though this trend was later shown to reverse in more complex logical problems (Trippas et al., 2017), the findings still brought evidence that people automatically process logical validity of inferences even under conditions in which they are asked to only judge their believability. Such autonomous manner of logical processing is again largely incompatible with the traditional default interventionist view (although, see Evans, 2018). 
Crucially, using the same interference paradigm, a very recent study by Thompson et al. (2018) showed that the extent to which logical processing interferes with the belief-based one is dependent on reasoners' cognitive capacity. That is, in highly cognitively disposed participants, logical processing seemed to have come more intuitively and thus interfered more with belief-based processing that vice versa. The opposite pattern was observed in less cognitively disposed participants who did show more interference by belief-based intuitive processing. This was true even though participants had to respond under strict deadline which limited their possibility to engage in analytic thought. Based on these findings, Thompson et al. (2018) concluded that there are indeed individual differences in intuitive processing such that for participants with higher cognitive dispositions, logical processing may come more intuitively than belief-based one. Also, the authors argued that these individual differences in intuitive logical processing may explain a part of the link between cognitive measures and reasoning performance which were previously attributed to the efficiency and disposition to engage in type 2 processing (Evans \& Stanovich, 2013; Stanovich, 2009).

The final line of evidence relevant to the purpose of the present research comes from studies which adopted a two-response paradigm (Thompson, Prowse Turner, \& Pennycook, 2011) to tease apart intuitive and analytic cognitive processes by presenting participants twice with the same reasoning problem. At the initial response stage, participants are instructed to respond intuitively and they are given a strict time limit or concurrent working memory load to restrict their possibility for analytic engagement. Subsequently, they are allowed unlimited time to affirm or rethink their initial answer and provide a final response. As was shown in two studies by Bago and De Neys (2017, 2019), participants were able to provide a normatively correct response in approximately $30 \%$ of conflict reasoning problems already at the initial response stage. This was true even when their possibility for analytic engagement was reduced as much as possible by the simultaneous use of instructions to respond intuitively, restricted time limit, and concurrent working memory load. Moreover, Bago and De Neys (2019) showed that changes in logical structure of the reasoning problem influenced people's initial answers and their conflict sensitivity, which is again incompatible with the view that reasoners only process the logic of the problem after engaging in type 2 thinking (for a similar finding, see also Newman, Gibb, \& Thompson, 2017). Lastly, in an earlier study using the two-response paradigm with several heuristics and biases problems, Thompson and Johnson (2014) showed that the accuracy of both initial and final answers was related to individual differences in cognitive ability, and to some extent also to actively open-minded thinking 
disposition. Thus, reasoners with a higher level of these cognitive factors were not only more accurate in solving reasoning problems when they had enough time for analytic thought, but also under conditions when their responding relied primarily on intuitive type 1 processing.

Over the time, these results have led many researchers to abandon the traditional defaultinterventionist model and formulate alternative hybrid dual-process accounts (De Neys, 2018; Pennycook, 2018; Thompson \& Newman, 2018; Trippas \& Handley, 2018; but see Evans, 2018). While there are certain differences between such accounts as proposed by various authors, all hybrid models incorporate a core assumption that multiple intuitive responses are cued by different aspects of the task at early stages of reasoning. That is, not only heuristic processing takes place intuitively, but people are also able to intuitively process the logic of the task they face, especially if it requires drawing relatively simple logical inferences (Evans, 2018). These intuitive answers differ in their speed or strength and relative differences between them will determine whether the reasoner experiences a conflict and chooses to engage in subsequent analytic thought to inhibit or rationalize one of the cued responses in favor of the other (e.g., Pennycook, 2018; Pennycook et al., 2015). Importantly, under the hybrid dual-process models, the strength or speed of intuitive logical processing is thought to not only depend on the characteristics of the task, i.e. its logical complexity, but also on individual differences between reasoners (Thompson \& Newman, 2018; Thompson et al., 2018). For example, it has been suggested that people who possess highly automatized mindware to answer the problem at hand may intuitively process its logical structure, efficiently detect the conflict, and give a normatively correct response with little need for engaging in more demanding analytic processing (Stanovich; 2018). Thus, whereas factors such as cognitive ability, thinking dispositions, numeracy, and/or cognitive reflection were traditionally thought to be related to engagement and efficiency of analytic thought (Evans \& Stanovich, 2013; Stanovich, 2009), an important novel aspect of hybrid models is the possibility for these individual differences to take place already at the initial processing stage.

In the present study, we set out to examine the contributions of several individual difference predictors to the reasoning accuracy at the intuitive and final reasoning stage using the tworesponse paradigm. This would allow us to tease apart the predictors of individual differences in intuitive logical processing from those factors that only contribute to reasoning performance when people have enough time to engage in analytic thought. To this end, we employed standard individual difference measures - i.e. cognitive ability, thinking dispositions, and cognitive reflection - which have been repeatedly shown to contribute to the 
accuracy on various reasoning problems (Toplak et al., 2011). Moreover, we also included indicators of participants' mindware instantiation and conflict detection efficiency, as these are theoretically regarded as the key determinants of sound reasoning (Pennycook et al., 2015; Stanovich, 2018), and have been recently empirically shown to substantially contribute to reasoning accuracy over and above the standard predictors (Šrol \& De Neys, 2019). We believe that examining the individual difference predictors of reasoning accuracy using the two-response paradigm could elucidate the diversity of cognitive processes that underlie intuitive and analytic stage of the reasoning process and thus advance the specification of current hybrid dual-process models.

\section{METHOD}

\section{Participants}

The study was run as an online survey and participants were students and alumni recruited through websites of major Slovak universities and colleges. We collected data from 149 participants (122 female, 27 male, age: $M=23.93, S D=4.50)^{1}$. Most of the participants reported having at least some college or university degree $(56 \%)$, the rest reported having finished high school education (44\%). The sample size was not determined beforehand but instead, we decided to collect as much data as possible in the time allocated for the data collection. No data were analyzed before the data collection was complete. Sensitivity power analysis carried out in G*Power 3.1.9 software (Faul, Erdfelder, Lang, \& Buchner, 2007) showed that the present sample size should provide at least $80 \%$ statistical power with $5 \%$ error probability to detect any correlations of $r>.227$, paired comparisons of Cohen's $d>$ 0.231 , and regression effect sizes of $f^{2}>.053$, for a single regression coefficient in a model with six predictors.

\section{Reading pretest}

Prior to the main study, we run a pilot to determine the reading time for syllogistic and baserate neglect problems for the use in our primary investigation. Using an online survey created

\footnotetext{
${ }^{1}$ The study contained two attention check questions and it was determined beforehand that the participants who will get both of them wrong will be automatically excluded from all analyses. However, as none of the responders failed to answer both of the questions, all participants were retained for the study.
} 
through Qualtrics we recruited 27 participants (16 female, 11 male, age: $M=23.59, S D=$ 1.95). They were presented with all 16 conflict and no-conflict syllogistic and base-rate neglect problems to be used in the main study. Reading time was measured as the latency from the onset of problem presentation until participants indicated that they have read the respective item by clicking a submit button at the bottom of the page. At the end of the pilot, participants were asked to confirm that they only read the items and did not attempt to solve them. All participants but one responded affirmatively to this, reading data from the one participant who did not were excluded from the analyses. Mean reading time was $4.60(S D=$ 1.28) for syllogisms and $6.30(S D=2.06)$ for base-rate neglect problems. These times were rounded to five and seven seconds, respectively, to give participants in the main study some minimal additional time to select their response.

\section{Materials}

\section{Reasoning problems presented under the two-response paradigm:}

We used two types of reasoning tasks in our study - syllogistic reasoning and base-rate neglect problems. All problems were presented under the two-response paradigm (e.g., Bago \& De Neys, 2017; Thompson et al., 2011). Every problem was displayed on a separate page, with the whole wording of the task showed to participants at the onset of the problem presentation. Both syllogistic and base-rate neglect tasks were preceded by specific instructions and an example problem intended to familiarize participants with the task at hand. Participants were informed that every problem will be presented to them two times. At the initial response stage, they were instructed to provide a first intuitive response that came to their minds after reading the problem under a strict time limit, i.e. five seconds for syllogisms and seven seconds for base-rate neglect items. The time restriction was previously shown to be an effective manipulation to knock out type 2 processing (Bago \& De Neys, 2017). During this phase, there was a timer presented on a page along with the reasoning problem that counted down the seconds to stress participants to answer within the time limit. If participants did not manage to choose an answer and submit their response in time, the experiment automatically moved to the next page. In such cases, all data regarding the participants' initial response, i.e. response accuracy, latency, and confidence were excluded from subsequent analyses. At the final response stage, participants were presented with the same problem but were allowed unlimited time to either rethink or affirm their answer and enter the final response. Additionally, at both times participants were also asked to indicate their confidence 
in the provided answer (see further). For both syllogisms and base-rate neglect tasks, participants first went through one practice problem to get familiar with the two-response paradigm and confidence measurement. The full wording of all reasoning problems used in the study is given in Section B of the supplementary material.

Syllogistic reasoning problems. In the syllogistic reasoning task, participants are presented with three statements - two premises and a conclusion - and are asked to decide whether the conclusion necessarily follows from the premises or not. We constructed four conflict syllogisms worded in a way that the believability of their conclusion was in conflict with its logical validity (i.e. syllogism was either valid but unbelievable, or invalid but believable). Four no-conflict items were then designed to be as similar in the problem content to conflict syllogisms as possible (see De Neys, Moyens, \& Vansteenwegen, 2010), but crucially, to contain conclusions in which the believability and logical validity was aligned (i.e. syllogisms were either valid and believable, or invalid and unbelievable). Four conflict items showed relatively good reliability both at the initial $(\alpha=.67)$, and final response stage $(\alpha=.78)$.

Base-rate neglect problems. We adopted problems based on rapid-response paradigm tasks similar to the ones used by Bago and De Neys (2017). In every problem, participants were given two pieces of information pertaining to an individual who was randomly drawn from among a sample which consisted of two groups (e.g. clowns and accountants). Firstly, participants were given a proportion of the two groups in the original sample ("There are 995 clowns and 5 accountants"). Then, they were presented with a single stereotypical trait pertaining to the individual, which cued one of the groups ("Person " $\mathrm{A}$ " is funny"). The task was to decide to which one of the groups the imaginary person more likely belongs to. Four no-conflict problems contained base-rate information which favored the group in line with the stereotypical trait, while four conflict items were created by simply changing the base-rates to favor the group contrary to the stereotypical characteristic. The conflict items showed good reliability both at the initial $(\alpha=.77)$, and final response stage $(\alpha=.78)$.

\section{Conflict detection indices:}

At every response stage, two measures were recorded which were intended to tap participants' conflict detection ability (Bago \& De Neys, 2017). First, for every reasoning problem, we measured the time from the onset of task presentation until participants selected a response and clicked on a submit button. This response latency was used in our analyses as a first conflict detection index. After submitting a response, participants were asked to indicate their 
confidence in their answer on a scale of 1 ("not at all confident") to 11 ("absolutely confident"). The response confidence was used as a second conflict detection index. Both indices were recorded at the initial, as well as the final, response stage.

\section{Mindware instantiation:}

As in previous research (Frey et al., 2018; Šrol \& De Neys, 2019), we used accuracy on neutral versions of reasoning problems as a measure of participants' mindware instantiation in these tasks. Two neutral syllogistic reasoning problems were used which were similar in their structure to conflict and no-conflict ones but did not have any actual content and consisted only of abstract statements (e.g. "All X are Y"). Two neutral base-rate neglect problems were also constructed to resemble conflict and no-conflict items but contained an attribute which did not cue any of the groups from which the individual was randomly chosen. Evidently, neutral tasks were designed specifically in such a way that they did not contain any intuitive cues to guide participants' reasoning. Therefore, neutral reasoning accuracy should primarily depend on the extent of participants' mindware knowledge necessary to solve the syllogistic and base-rate neglect problems, i.e. the rules of logical inference and the impact of base-rates on the probabilistic judgment.

Average accuracy on the four neutral reasoning tasks ${ }^{2}$ was high $(M=79 \%, S D=18.47)$. As was argued by Stanovich (2018), mindware instantiation is rather task specific and in line with this we have observed very low reliability for the four neutral reasoning problems $(\alpha=$ -.04). However, when we redid the analyses with neutral syllogistic reasoning and base-rate neglect items separately, the results were largely consistent with the ones obtained by using a single composite mindware instantiation measure from both types of tasks combined. Therefore, for simplicity, we have decided to retain the single mindware instantiation index computed from the four neutral problems. We should note that the mindware index showed theoretically predicted and empirically meaningful relationships with reasoning performance and conflict detection ability which is why we believe its use is justified despite the low internal consistency (see also Šrol \& De Neys, 2019).

Standard individual difference measures:

\footnotetext{
${ }^{2}$ Although we did not intend to analyse neutral problem accuracy at the first and second response stage, problems were presented to participants under the two-response paradigm to ensure similarity with the presentation format of other reasoning tasks. In all analyses regarding neutral problems, we report the accuracies observed at the final response stage.
} 
The descriptive statistics for individual difference measures are reported in Table 1.

Cognitive ability. We used a shortened, 9-item version of the Vienna Matrix Test (Klose, Černochová, \& Král, 2002) to measure participants' cognitive ability. The test resembles Raven's progressive matrices in that the items consist of $3 * 3$ picture matrix with one tile missing and participants need to find a pattern and complete the figure by choosing one of the eight response alternatives. Originally, the Czech adaptation of the test by Klose et al (2002), upon which the present method is based, consists of 24 items of increasing difficulty. For the purpose of the present study, we used a 9-item version based on the previous study by Šrol (2019), who showed that the short measure shows good composite reliability $(\alpha=.76)$ and a very high correlation with the original test $(r=.92)$. The shortened version was chosen here to reduce participants' burden due to the overall length of the materials used in the study.

Need for Cognition (NFC). To tap participants' analytic thinking disposition, we used a 5item version of the Need for Cognition scale (Study 2, Epstein, Pacini, Denes-Raj, \& Heier, 1996). An example item was: "I prefer complex to simple problems". Participants were asked to rate their agreement with the items using a scale from 1 ("completely disagree") to 5 (“completely agree"). Average rating on the five items was used as a measure of participants' inclination toward analytic thought.

Faith in Intuition (FI). Intuitive thinking disposition was measured by a 5-item Faith in Intuition scale taken from the second study reported by Epstein et al. (1996). Participants were asked to rate their agreement with the items (an example item: "I believe in trusting my hunches") using the same scale as in the case of Need for Cognition. The ratings on the five items were averaged to produce a measure of the tendency toward intuitive thought.

Cognitive reflection. Seven items which evoked misleading intuitive responses were used to measure cognitive reflection. These included the original three problems from the Frederick's (2005) test, although with changed content and numbers (e.g. "If it takes 3 printers 3 minutes to print 3 newspapers...”), as this simple manipulation has been shown to reduce the effect of possible familiarity with the original test items (Chandler, Mueller, \& Paolacci, 2014). Four additional problems were taken from previous research (Šrol, 2019). The sum of correct answers on the seven items was used as an indicator of participants' cognitive reflection.

\section{Procedure}


The study was run as an online survey created in Qualtrics software. Participants were first presented with an informed consent and filled in several demographic questions. The remainder of the study consisted of two blocks of materials, one containing the reasoning problems presented under the two-response paradigm and the other consisting of the standard individual difference measure and one additional measure which is not reported here. The order of the blocks was randomized between participants and the order of materials within each block was randomized within participants. Conflict and no-conflict versions of reasoning problems were presented to participants in random order, but neutral versions of both tasks were always presented last so as to not influence participants subsequent responses (Frey et al., 2018). Participants were not explicitly told their responses to reasoning problems were timed but were instructed not to take breaks while solving these problems and to submit their answers immediately after deciding on the response. Every standard individual difference measure was presented on a separate page along with instructions with the exception of Need for Cognition and Faith in Intuition scales, which were intermixed on a single page and participants rated them using the same response scale.

Table 1. Descriptives of individual difference measures

\begin{tabular}{lccc}
\hline & $M$ & $S D$ & $\alpha$ \\
\hline Cognitive ability & 5.37 & 1.96 & .62 \\
Need for Cognition & 3.66 & 0.77 & .77 \\
Faith in Intuition & 3.37 & 0.79 & .80 \\
Cognitive reflection & 4.07 & 1.80 & .70 \\
\hline Note. The table contains & mean & scores, standard \\
deviations, and reliability estimates (Cronbach's $\alpha$ ) for \\
standard individual difference measures in the study.
\end{tabular}

\section{RESULTS}

The main aim of the present study was to examine standard individual difference measures, mindware instantiation, and conflict detection efficiency as predictors of participants' accuracy in conflict reasoning problems at the initial and final response stage. We, however, first present the results of traditional group-level conflict detection (Frey et al., 2018; Mevel et al., 2014; Pennycook et al., 2015) and direction of change analyses (Bago \& De Neys, 2017) for comparability with previous research and sketch our approach to estimating participants' overall detection efficiency from several detection indices combined (Frey \& De Neys, 2017; Šrol \& De Neys, 2019). Then, we explore the correlations between standard individual 
difference measures, mindware instantiation, detection efficiency, and reasoning accuracy, i.e. overall accuracy on conflict reasoning problems, at the initial and final response stage. Finally, we present two regressions to examine our individual difference measures as independent predictors of reasoning accuracy separately at the two response stages.

\section{Group-level analysis of reasoning accuracy, direction of answer change, and conflict detection}

A general overview of the group-level results for reasoning accuracy and conflict detection analyses is presented in Table 2 . We examined the results for both reasoning tasks separately, but for simplicity, we have also calculated overall accuracy, response latency, and confidence combined for all eight reasoning problems, i.e. both syllogisms and base-rate tasks. As there was a restricted time limit at the initial response stage for all problems, those cases when participants did not manage to meet the deadline, their accuracy, latency, and confidence for the response were excluded from all analyses ${ }^{3}$. Participants' accuracy on no-conflict problems was much higher than on the conflict ones both at the initial and final response stage, $t$ 's $>$ $14.00, p$ 's $<.001, d$ 's $>1.15$. Importantly, while the conflict accuracy was higher at the time of the final response, as would be predicted by the traditional dual-process accounts (e.g. Evans \& Stanovich, 2013), participants actually managed to solve $44 \%$ of conflict reasoning problems accurately already at the initial response stage. Relatively high initial conflict reasoning accuracy has been observed also by Bago and De Neys (2019). Overall performance results are completely consistent with the accuracies observed separately on both reasoning tasks.

Besides analysing participant's accuracies at the initial and final response stage, the use of the two-response paradigm allows us to examine the specific directions in which people changed their initial answers after having unrestricted time to engage in analytic processing. Following the direction of change analyses presented by Bago and De Neys (2017, 2019), we have categorized participant's responses on reasoning problems into four possible directions of answer change: correct initial and correct final answer ("11" category), incorrect initial and incorrect final answer (" $00 "$ category), correct initial and incorrect final answer ("10"

${ }^{3}$ Over the 16 conflict and no-conflict syllogisms and base-rate neglect problems, this amounted to 244 responses (approximately 10\% of all responses). This gives an indication that the time limit was effective and it was not easy for participants to meet the deadline. Similar percentage of missing responses due to time restriction was reported by Bago and De Neys $(2017,2019)$. 
category) or incorrect initial and correct final answer ("01” category). The frequencies of these direction of change categories for conflict and no-conflict syllogisms and base-rate neglect problems are presented in Table 3.

Table 2. Summary of the group-level analysis of reasoning accuracy and conflict detection

\begin{tabular}{|c|c|c|c|c|c|c|}
\hline & \multicolumn{2}{|c|}{ Accuracy } & \multicolumn{2}{|c|}{ Response latency } & \multicolumn{2}{|c|}{ Response confidence } \\
\hline & Initial & Final & Initial & Final & Initial & Final \\
\hline Syllogistic reasoning $(n)$ & 148 & 149 & 114 & 99 & 114 & 99 \\
\hline no-conflict $(S D)$ & $85 \%(20.08)$ & $87 \%(20.26)$ & $4.46(0.56)$ & $6.74(4.46)$ & $9.72(1.46)$ & $10.06(1.20)$ \\
\hline conflict $(S D)$ & $48 \%(36.17)$ & $59 \%(36.64)$ & $4.44(0.58)$ & $7.26(5.95)$ & $8.87(2.28)$ & $9.84(1.60)$ \\
\hline difference & $t(147)=10.77$ & $t(148)=8.78$ & $t(113)=0.31$ & $t(98)=0.89$ & $t(113)=4.41$ & $t(98)=1.52$ \\
\hline$p$-value & $<.001$ & $<.001$ & .754 & .376 & $<.001$ & .132 \\
\hline Cohen's $d$ & $d=0.89$ & $d=0.72$ & $d=0.03$ & $d=0.09$ & $d=0.41$ & $d=0.15$ \\
\hline Base-rate neglect $(n)$ & 149 & 149 & 119 & 110 & 119 & 110 \\
\hline no-conflict $(S D)$ & $95 \%(15.78)$ & $96 \%(11.89)$ & $5.62(0.91)$ & $6.13(3.31)$ & $9.39(1.90)$ & $9.73(1.74)$ \\
\hline conflict $(S D)$ & $41 \%(38.16)$ & $51 \%(38.27)$ & $5.87(1.02)$ & $8.16(7.06)$ & $8.24(2.14)$ & $8.53(2.13)$ \\
\hline difference & $t(148)=15.52$ & $t(148)=13.64$ & $t(118)=3.08$ & $t(109)=3.81$ & $t(118)=6.73$ & $t(109)=6.70$ \\
\hline$p$-value & $<.001$ & $<.001$ & .003 & $<.001$ & $<.001$ & $<.001$ \\
\hline Cohen's $d$ & $d=1.27$ & $d=1.12$ & $d=0.28$ & $d=0.36$ & $d=0.62$ & $d=0.64$ \\
\hline Overall performance $(n)$ & 149 & 149 & 137 & 131 & 137 & 131 \\
\hline no-conflict $(S D)$ & $90 \%(13.47)$ & $91 \%(12.42)$ & $5.11(0.69)$ & $6.71(3.27)$ & $9.62(1.36)$ & $9.98(1.13)$ \\
\hline conflict $(S D)$ & $44 \%(29.28)$ & $55 \%(30.19)$ & $5.24(0.85)$ & $7.66(5.56)$ & $8.62(1.87)$ & $9.07(1.91)$ \\
\hline difference & $t(148)=17.14$ & $t(148)=14.00$ & $t(136)=1.87$ & $t(130)=2.35$ & $t(136)=7.35$ & $t(130)=6.15$ \\
\hline$p$-value & $<.001$ & $<.001$ & .063 & .020 & $<.001$ & $<.001$ \\
\hline Cohen's $d$ & $d=1.40$ & $d=1.15$ & $d=0.16$ & $d=0.21$ & $d=0.63$ & $d=0.54$ \\
\hline
\end{tabular}

Note. Overall performance reflects participants' mean accuracy, response latency, and confidence at the two response stages averaged across all eight conflict reasoning problems. Response latency data are reported in seconds. Cohen's $d$ is reported as a measure of effect size. Significant paired differences are presented in bold $(p<.05)$.

As can be seen from the table, two response patterns had very high and similar prevalence on the crucial conflict reasoning problems, i.e. " 00 " and " 11 " answers. The high percentage of " $00 "$ responses is quite in line with the traditional default-interventionist model (e.g. Evans \& Stanovich, 2013), as it shows that people tend to stick with their initial incorrect response, presumably due to the failure to inhibit the compelling heuristic answer. On the other hand, the second response pattern which would be predicted on the basis of default-interventionist account would presume that after an incorrect intuitive response people engage in type 2 process intervention and correct their initial answer. However, this direction of answer change, i.e. the " 01 " category, was shown to be relatively rare in participant's responses to conflict problems. In fact, the majority of correct final answers observed on the conflict reasoning problems were correct already at the initial response stage, as indicated by the high proportion of "11" responses. Our results in this regard replicate the findings of Bago and De 
Neys (2017, 2019), who similarly showed that, in contrast with the default-interventionist model, people are often capable of producing correct answers already at the initial response stages presumably through the logical type 1 processing, supporting this aspect of the hybrid dual-process model.

Table 3. Direction of change analysis for conflict and no-conflict reasoning problems

\begin{tabular}{llcccc}
\hline \multirow{2}{*}{ Task } & \multicolumn{4}{c}{ Direction of change } \\
\cline { 3 - 6 } & & 11 & 00 & 01 & 10 \\
\hline Base-rate & Conflict & $35.8 \%(197)$ & $43.6 \%(240)$ & $14.9 \%(82)$ & $5.6 \%(31)$ \\
neglect & No-conflict & $93.5 \%(520)$ & $1.6 \%(9)$ & $2.9 \%(16)$ & $2.0 \%(11)$ \\
\hline Syllogistic & Conflict & $44.1 \%(226)$ & $40.4 \%(207)$ & $12.3 \%(63)$ & $3.3 \%(17)$ \\
reasoning & No-conflict & $83.4 \%(437)$ & $9.2 \%(48)$ & $6.1 \%(32)$ & $1.3 \%(7)$ \\
\hline
\end{tabular}

Note. The table contains frequencies and percentages of trials on which a specific direction of answer change for conflict and no-conflict syllogisms and base-rate neglect problems was observed. Direction of change categories reflect: "11" = correct initial and correct final response; " $00 "=$ incorrect initial and incorrect final response; " $01 "=$ incorrect initial and correct final response; "10" = correct initial and incorrect final response.

Next, we have calculated average response latency ${ }^{4}$ and confidence for conflict and noconflict reasoning problems at both response stages to see whether we would observe the traditional conflict detection effects, i.e. longer response latency and lower confidence on the incorrectly solved conflict in comparison with correctly solved no-conflict problems. Following previous conflict detection studies (e.g., Frey et al., 2018; Mevel et al., 2014), participants who gave no correct answers on no-conflict or incorrect answers on conflict problems were dropped from the respective analyses ( $n$ 's indicated in Table 2). For simplicity, we have again computed overall response latency and confidence for all eight reasoning problems combined, but analyses for the two reasoning tasks separately are also presented in Table 2. At the final response stage, participants on a group-level showed traditional conflict detection signs, i.e. they took longer to produce an answer to incorrectly solved conflict than correctly solved no-conflict problems, however, this difference was more modest and did not reach significance at the first response stage. Considering the second conflict detection index, participants reported lower response confidence after the incorrectly solved conflict than correctly solved no-conflict reasoning problems both at the first and second response stage, $t$ 's

\footnotetext{
${ }^{4}$ Prior to the analyses, we have checked the response latency data for outlying observations. Any latencies which lied more than three standard deviations from the mean of the respective variable were windsorized to the value of mean $+/$ - three standard deviations.
} 
$>6.15, p$ 's $<.001, d$ 's $>0.54$. Therefore, as was argued previously, people do indeed seem to be sensitive to conflict during reasoning even under conditions which are designed to reduce the involvement of type 2 processing (Bago \& De Neys, 2017; Franssens \& De Neys, 2009; Johnson et al., 2016).

However, as can be seen from the table, conflict detection findings are driven mostly by baserate neglect items, among which the comparisons for both latency and confidence between conflict and no-conflict tasks were significant at either response stage. In syllogisms, we found no significant differences in the case of response latency index. Weaker conflict detection effects in syllogistic reasoning task in comparison with other reasoning problems have been reported previously (Šrol \& De Neys, 2019), and they likely reflect the fact that the conflict in syllogisms is not as explicitly present as, for example, in base-rate problems with extreme ratios. This would be consistent with recent theorizing that the strength of logical and heuristic intuitions are likely task specific (Thompson \& Newman, 2018). While extreme base-rates are easily processed and give rise to quick and fluent logical intuition which conflicts with the stereotypical description of an individual in this task, in more complex logical problems, such as base-rate tasks with moderate ratios or syllogisms, logical type 1 response may not come as quickly and fluently (Pennycook, Fugelsang, \& Koehler, 2012; Trippas et al., 2017). And yet, interestingly, there was a significant conflict detection effect on syllogisms in the case of response confidence index at the initial response stage. Thus, it would seem that at least some rudimentary logical processing may come quickly enough even in syllogisms to give rise to a conflict with belief-based intuition.

\section{Estimating participants' conflict detection efficiency}

Following recent studies on individual differences in conflict detection (Frey \& De Neys, 2017; Šrol \& De Neys, 2019), we have adopted an approach to estimate participants' ability to consistently detect conflicts across different reasoning problems and detection indices. To do this, we have calculated detection efficiency for every participant, as the sum of indices on which participant showed successful detection (i.e. longer response latency or lower confidence on the incorrect conflict in comparison with correct no-conflict problem) divided by the total number of times participant was biased ${ }^{5}$. Participants who were not biased on any

\footnotetext{
${ }^{5}$ It is necessary to take into account not only how many times one shows a successful detection either by having longer response latency or lower confidence, but also how many times they were biased on a particular conflict reasoning task and responded incorrectly. As
} 
of the reasoning problems were excluded from the analyses pertaining to detection efficiency indices (initial response: $n=12$, final response: $n=18$, overall: $n=11$ ).

Firstly, we have calculated detection efficiency separately for the detection indices observed at the two response stages. However, participants did not differ in their detection efficiency at the time of their initial $(M=0.53, S D=0.32, \alpha=.30)$ and final response $(M=0.55, S D=$ $0.32, \alpha=.36), t(129)=0.50, p=.616, d=0.04$, and the two efficiencies were moderately correlated, $r=.39, \mathrm{p}<.001$. Therefore, for simplicity we have decided to compute overall conflict detection efficiency $(M=0.56, S D=0.26, \alpha=.48)$ from all reasoning tasks and detection indices at both response stages combined. This composite was used as an indicator of conflict detection ability in all subsequent analyses ${ }^{6}$.

As can be seen from the overall detection efficiency, participants successfully detected more than half of the conflicts on the tasks on which they were biased. Such relatively high prevalence of successful detection was also observed in previous studies which report individual-level conflict detection analyses (Frey et al., 2018; Mevel et al., 2014; Pennycook et al., 2015; Šrol \& De Neys, 2019). Interestingly, participants' detection efficiency is remarkably similar at the initial and final response stage, suggesting that sensitivity to conflict takes place very early in the reasoning process (Bago et al., 2018). This again converges with the conclusions of previous studies which suggested that conflict detection is already successful at intuitive problem processing stage (Bago \& De Neys, 2017; Franssens \& De Neys, 2009; Johnson et al., 2016), and additional time for the engagement of analytic resources does not seem to further improve the ability to detect conflict during reasoning.

Individual differences in reasoning accuracy at the initial and final response stage

the cases when participants give correct responses to conflict tasks are not included in conflict detection analyses (see above), these instances have to be excluded when calculating participants' detection efficiency. For this reason, to obtain a measure of how efficient participants were at detecting conflict, one has to divide the sum of successfully detected conflicts by the total number of times participants were biased and responded incorrectly on the conflict reasoning problems (see also Šrol \& De Neys, 2019)

${ }^{6}$ As can be seen from the results above, the detection efficiency index is of relatively low internal consistency. While some caution is certainly necessary when interpreting the detection efficiency analyses here, we note that the results presented below are completely consistent with previous empirical research on individual differences in conflict detection (Šrol \& De Neys, 2019). 
We now move on to the key aim of the present study, i.e. examining individual differences in reasoning performance at the two response stages. As the first step in the analyses, we have calculated correlations between standard individual difference measures, mindware instantiation, detection efficiency, and reasoning accuracy (i.e. overall reasoning performance) at the initial and final response stage. Reasoning accuracy was calculated as an average conflict reasoning performance on both syllogisms and base-rate neglect problems combined separately at the initial $(M=44 \%, S D=29.28, \alpha=.78)$ and final response stage $(M$ $=55 \%, S D=30.19, \alpha=.78)$. The results of the correlation analysis are presented in Table 4 .

Table 4. Correlations between the initial and final reasoning accuracy, standard individual difference measures, mindware instantiation, and conflict detection efficiency

\begin{tabular}{lccccccc}
\hline & 1. & 2. & 3. & 4. & 5. & 6. & 7. \\
\hline 1. Initial reasoning accuracy & 1 & & & & & & \\
2. Final reasoning accuracy & $\mathbf{. 8 3}$ & 1 & & & & & \\
3. Cognitive ability & $\mathbf{. 2 3}$ & $\mathbf{. 3 4}$ & 1 & & & & \\
4. Cognitive reflection & $\mathbf{. 4 0}$ & $\mathbf{. 4 3}$ & $\mathbf{. 3 5}$ & 1 & & & \\
5. Need for Cognition & .11 & .13 & .08 & $\mathbf{. 2 0}$ & 1 & & \\
6. Faith in Intuition & .03 & .02 & -.02 & .04 & .14 & 1 & \\
7. Mindware instantiation & $\mathbf{. 2 9}$ & $\mathbf{. 3 2}$ & $\mathbf{. 2 0}$ & $\mathbf{. 2 0}$ & -.05 & .09 & 1 \\
8. Detection efficiency & $\mathbf{. 2 5}$ & $\mathbf{. 3 0}$ & .12 & $\mathbf{. 3 5}$ & -.03 & -.09 & $\mathbf{. 1 9}$ \\
\hline
\end{tabular}

Note. Correlations with detection efficiency are based on 138 observations, others are based on 149 observations. Correlations that appear in bold are significant at $p<.05$.

Focusing first on the standard individual difference measures, these show remarkably similar patterns of correlations with reasoning accuracy observed at the initial and final response stage. Specifically, both reasoning accuracies are moderately positively related to cognitive ability and cognitive reflection. The correlations with cognitive ability are roughly similar to those reported by Thompson and Johnson (2014), however, these authors also found an association with an actively open-minded thinking disposition, although only in case of first responses to base-rate neglect, but not syllogistic reasoning tasks. Here, we observed a trend for Need for Cognition to be positively related to the initial and final reasoning accuracy, however, in neither case was it statistically significant. Faith in Intuition, on the other hand, seemed to be completely unrelated to reasoning performance. While the correlations with cognitive ability and cognitive reflection seem to be a bit stronger in case of final reasoning accuracy that the initial one, the differences are minute. Overall, the correlational results seem to suggest that the same cognitive factors that are argued to determine the efficiency of 
analytic thought (e.g., Šrol \& De Neys, 2019; Toplak et al., 2011) might be associated with reasoning performance even under circumstances when type 2 processing is restricted (Thompson et al., 2018). Moreover, reasoning accuracy under time pressure is very highly correlated with the one observed after allowing participants unlimited time to rethink or affirm their initial response, further suggesting that the factors which determine reasoning accuracy under both conditions might be relatively similar.

Moving on to the associations with mindware instantiation and detection efficiency, these again show completely consistent correlations with reasoning accuracy at the initial and final response stage. In line with the theoretical predictions (De Neys \& Bonnefon, 2013; Pennycook et al., 2015; Stanovich et al., 2016), and recent empirical evidence for the role of mindware and conflict detection as key determinants of sound reasoning performance (Šrol \& De Neys, 2019), both of these variables were moderately positively correlated with reasoning accuracy. These results seem to fit especially well with the recent theorizing by Stanovich (2018), who argued that for people with highly automatized mindware, normatively correct responses on reasoning problems may come with such high level of activation that they facilitate relatively effortless conflict detection, and may actually dominate over heuristic processing without almost any need for analytic engagement (see also De Neys, 2018; Pennycook, 2018). Correlations of mindware instantiation and detection efficiency with initial reasoning accuracy seem to be highly compatible with such a view. Also notably, as in a recent study by Šrol and De Neys (2019), we have found conflict detection efficiency to be related to differences in participants' mindware instantiation and cognitive reflection.

\section{Predicting reasoning accuracy at the initial and final response stage}

Finally, to examine the standard individual difference measures, mindware instantiation, and detection efficiency as independent predictors of reasoning accuracy, we have conducted two regression analyses for reasoning accuracy at the initial and final response stage. In both analyses, we have entered the variables into the regression in three steps. In line with their roles as key components of reasoning accuracy (De Neys \& Bonnefon, 2013; Pennycook et al., 2015; Stanovich, 2018), we have entered mindware instantiation and detection efficiency at the first two steps of regression. Then, in the final model, we entered all standard individual difference measures into the model to examine whether they predict any additional variance in 
initial and final reasoning accuracy over and above mindware instantiation and detection efficiency. The results for both regressions are summarized in Table $5 .^{7}$

Table 5. Summary of the regression analyses predicting reasoning accuracy at the initial and final response stage

\begin{tabular}{|c|c|c|c|c|c|c|c|c|}
\hline & \multicolumn{4}{|c|}{ Initial reasoning accuracy } & \multicolumn{4}{|c|}{ Final reasoning accuracy } \\
\hline & $b(S E)$ & $\beta$ & $t$ & $p$ & $b(S E)$ & $\beta$ & $t$ & $p$ \\
\hline \multicolumn{9}{|l|}{ Step 1} \\
\hline Constant & $0.12(0.09)$ & & 1.29 & .201 & $0.15(0.10)$ & & 1.50 & .135 \\
\hline \multirow[t]{2}{*}{ Mindware } & $0.36(0.12)$ & .26 & 3.10 & .002 & $0.46(0.13)$ & .30 & 3.65 & $<.001$ \\
\hline & \multicolumn{4}{|c|}{$R^{2}=.059, F(1,136)=9.58, p=.002$} & \multicolumn{4}{|c|}{$R^{2}=.083, F(1,136)=13.35, p<.001$} \\
\hline \multicolumn{9}{|c|}{ 然 } \\
\hline Constant & $0.05(0.10)$ & & 0.52 & 602 & $0.06(0.10)$ & & 0.60 & .549 \\
\hline Mindware & $0.30(0.12)$ & .22 & 2.61 & .010 & $0.39(0.13)$ & .25 & 3.10 & .002 \\
\hline \multirow[t]{2}{*}{ Detection efficiency } & $0.20(0.08)$ & .21 & 2.52 & .013 & $0.27(0.09)$ & .25 & 3.04 & .003 \\
\hline & \multicolumn{4}{|c|}{$\Delta R^{2}=.042, F(1,135)=6.34, p=.013$} & \multicolumn{4}{|c|}{$\Delta R^{2}=.058, F(1,135)=9.22, p=.003$} \\
\hline \multicolumn{9}{|l|}{ Step 3} \\
\hline Constant & $-0.09(0.16)$ & & -0.59 & .556 & $-0.18(0.17)$ & & -1.11 & .269 \\
\hline Mindware & $0.24(0.12)$ & .18 & 2.10 & .038 & $0.30(0.12)$ & .20 & 2.47 & .015 \\
\hline Detection efficiency & $0.13(0.08)$ & .13 & 1.50 & .136 & $0.18(0.09)$ & .17 & 2.00 & .047 \\
\hline Cognitive ability & $0.01(0.01)$ & .09 & 1.00 & .322 & $0.03(0.01)$ & .21 & 2.48 & .014 \\
\hline Analytic thinking disp. & $0.01(0.03)$ & .02 & 0.28 & .781 & $0.02(0.03)$ & .06 & 0.80 & .424 \\
\hline Intuitive thinking disp. & $0.00(0.03)$ & .01 & 0.14 & .886 & $-0.00(0.03)$ & -.01 & -0.08 & .940 \\
\hline \multirow[t]{2}{*}{ Cognitive reflection } & $0.03(0.01)$ & .22 & 2.39 & .018 & $0.03(0.01)$ & .20 & 2.21 & .029 \\
\hline & \multicolumn{4}{|c|}{$\Delta R^{2}=.066, F(4,131)=2.63, p=.037$} & \multicolumn{4}{|c|}{$\Delta R^{2}=.110, F(4,131)=4.84, p=.001$} \\
\hline
\end{tabular}

Note. The table contains unstandardized $(b)$ and standardized regression coefficients $(\beta)$ with their respective $t$-ratio and significance. $R^{2}$ and $\Delta R^{2}$ denote adjusted $r$-square for the initial model and change in $r$-square at the $2^{\text {nd }}$ and $3^{\text {rd }}$ step of the regression with appropriate change statistics. Significant regression coefficients are presented in bold. * $p<.05$; ** $p<.01 ; * * * p$ $<.001$

Considering first the responses under a time limit, both mindware instantiation and detection efficiency emerged as significant independent predictors of approximately the same strength and together accounted for $10 \%$ of the variance in initial reasoning accuracy. Again, this lends some credence to the notion that well-automatized mindware may be precisely the factor which makes some reasoners highly likely both to detect the conflict and to derive a

\footnotetext{
${ }^{7}$ For an interested reader, we have also complimented these analyses by a third regression in which we examined the same variables as predictors of the probability of "corrective answer change", i.e. the proportion of responses on which participants managed to change their initially incorrect answer on the conflict reasoning problems into a final correct response. The results are presented in the Section A of the supplementary material. Despite the differences of the two analytic approaches, the results presented there are relatively similar to the conclusions of two regressions presented in the main manuscript.
} 
normatively correct response even under conditions when type 2 processing is restricted (Stanovich, 2018). Entering standard individual difference predictors led to a substantial increase in the predictive power of the model, however, only cognitive reflection emerged as an independent predictor $(\beta=.22)$ from among these variables. Importantly, at the final step of the regression, detection efficiency no longer significantly contributed to initial reasoning accuracy $(\beta=.13, p=.136)$, although it was still positively related to it. This might be because cognitive reflection has been previously shown to tap conflict detection ability (Pennycook et al., 2014; Šrol \& De Neys, 2019), and was moderately correlated with detection efficiency here. Thus, it seems that it explained away some of the variance in initial reasoning accuracy which was accounted for by detection efficiency. This seems consistent with the notion of cognitive reflection trait as an indicator of people's sensitivity to situations when reliance on intuition may be insufficient and analytic processing is needed (Frederick, 2005; Stanovich et al., 2016). In contrast with the detection efficiency, mindware instantiation remained to contribute to initial reasoning accuracy $(\beta=.18)$ in the final model. Together the variables explained approximately $14 \%$ of the variance at the last step of the regression.

In the second analysis, both mindware instantiation and detection efficiency predicted final reasoning accuracy, but this time both remained significant independent predictors even at the last step of the regression. Entering standard individual difference measures led to a substantial increase in the predictive power of the model and both cognitive ability $(\beta=.21)$ and cognitive reflection $(\beta=.20)$ showed up to significantly contribute to reasoning accuracy at the final response stage over and above mindware instantiation and detection efficiency indices. Overall, the results are largely consistent with the findings of Srol and De Neys (2019), who likewise showed that a set of standard individual difference measures and indicators of mindware and detection independently contribute to accuracy in various reasoning problems. However, although both thinking dispositions were related to reasoning accuracy in the expected direction, neither one emerged as a significant independent predictor here. Together, the variables explained approximately $22 \%$ of the variance in the reasoning accuracy at final response stage.

\section{DISCUSSION}

In the present study, we examined how standard individual difference measures, mindware instantiation, and detection efficiency contribute to reasoning accuracy when people are asked 
to solve reasoning problems under a time limit, thus restricting their possibility to engage in analytic operations. First of all, we observed that while accuracy on conflict reasoning problems is indeed higher when people are allowed unlimited time to think about the task, on average reasoners managed to solve almost half of the problems correctly even when they were asked to give an intuitive response under a strict deadline. Conflict detection analyses suggested that already at the initial response stage, reasoners were sensitive to the conflict inherent in the heuristics and biases problems and that this sensitivity was not increased when they were allowed further time to rethink or affirm their initial response. Both findings are consistent with recent theorizing and empirical evidence which suggests that people engage in both logical and heuristic processing already at the intuitive stages of reasoning (for reviews, see De Neys, 2018; Pennycook, 2018; Thompson \& Newman, 2018; Trippas \& Handley, 2018). Crucially, however, we have shown that already at this initial reasoning stage, substantial individual differences between reasoners exist in reasoning accuracy and that these are related to their mindware instantiation, detection efficiency, and cognitive reflection. Moreover, we have also replicated some of the recent findings regarding the role of mindware instantiation and cognitive reflection as important determinants of the efficiency in conflict detection during reasoning (Šrol \& De Neys, 2019).

Historically, individual differences in reasoning accuracy related to variables such as cognitive ability, thinking dispositions, or cognitive reflection have all been attributed to the tendency toward analytic processing and the efficiency of this type of thought (e.g., Evans \& Stanovich, 2013a; Stanovich, 2009; Stanovich \& West, 2000). The results presented here suggest that at least part of the link between such cognitive dispositions and reasoning accuracy is actually due to differences in intuitive processing (Thompson et al., 2018). We have found initial reasoning performance to be mostly related to participants' mindware instantiation, cognitive reflection, and to some extent, detection efficiency. The contributions of these factors fit well with the recent account outlined by Stanovich and his colleagues (Stanovich, 2018; Stanovich et al., 2016), who emphasize the role of mindware instantiation as a key determinant of conflict detection efficiency and overall reasoning accuracy. According to their view, reasoners with highly automatized mindware knowledge may be capable of processing the logical structure of a problem at hand relatively intuitively, and thus their ability to detect the conflict and produce a normatively correct response comes without the need to engage in demanding analytic operations. Consistently with this, we have observed that mindware instantiation and detection efficiency are mutually correlated and that 
they not only both predict the final reasoning performance but are also among the few predictors which contribute to reasoning accuracy already at the intuitive processing stage. The role of cognitive reflection in initial reasoning accuracy is also not surprising, as it was shown previously to be moderately related to mindware instantiation and to predict detection efficiency independently of other individual difference measures (Šrol \& De Neys, 2019). This additionally suggests that cognitive reflection might be such a surprisingly strong predictor in reasoning and decision-making domains (e.g. Toplak et al., 2011) because it simultaneously taps into two of the key components of the susceptibility to cognitive biases numerical mindware knowledge and the ability to monitor for conflict during reasoning (see also Pennycook et al., 2014; Sinayev \& Peters, 2015).

Not surprisingly, we found that the proportion of variance accounted for by individual difference predictors was lower in case of initial reasoning performance than with the final one. Obviously, if one has little to no time to engage in analytic processing to inhibit heuristic intuition and derive a normatively correct response, the role of those cognitive variables which primarily determine the efficiency and extent of type 2 processing is largely limited. Accordingly, we have observed that cognitive ability, an indicator of the cognitive resources available for carrying out demanding type 2 processing such as response inhibition and hypothetical thinking (Evans \& Stanovich, 2013; Stanovich, 2009; Stanovich et al., 2016), predicted final, but not initial reasoning accuracy. In a similar vein, analytic thinking disposition was not found here to be related to initial reasoning accuracy, presumably as one can only make use of their tendency to process information analytically in situations when they have enough time approach the problem in such manner. And yet, as the thinking dispositions were also unrelated to final reasoning accuracy, we should refrain from drawing any strong conclusions about their role at the initial reasoning stage. We would mention, however, that Thompson and Johnson (2014) and Thompson et al. (2018) both found cognitive ability and actively open-minded thinking disposition to be related to individual differences in intuitive logical processing. Still, both of these measures have been previously shown also to correlate with mindware instantiation on several traditional heuristics and biases problems (Šrol \& De Neys, 2019). Therefore, it is currently not possible to distinguish whether it is mindware, as suggested by Stanovich (2018; Stanovich et al., 2016), or other cognitive predictors that primarily determine the efficiency of intuitive logical processing.

While the main focus of our study lied in the examination of individual difference predictors of initial reasoning accuracy, our design also made it possible to examine and replicate some 
of the recent findings on conflict detection during reasoning brought forward by Šrol and De Neys (2019). Specifically, consistently with results they report, we have found that detection efficiency is mostly related to participants' mindware instantiation and cognitive reflection and that it predicts reasoning performance independently of other individual difference measures. Interestingly, while our study was not specifically aimed at testing the comparisons of conflict detection at the two response stages, we did observe that participants' detection efficiency did not increase after they were allowed additional time to engage in analytic thought, but rather stayed approximately the same as it was already at the time of the initial response. This is in line with the evidence that conflict detection takes place early in the reasoning process (Bago et al., 2018) and is largely unaffected by the restrictions placed on type 2 processing (Bago \& De Neys, 2017; Franssens \& De Neys, 2009; Johnson et al., 2016).

However, it should also be noted that the use of the two-response paradigm may have influenced our conflict detection findings. For example, response latency detection results at the initial response stage may be substantially attenuated as there is very little room for reasoners to allow themselves more time to process the conflict problem when operating under a strict deadline (Bago \& De Neys, 2017). This might be precisely why we did not observe a group-level detection effect on syllogisms with response latency at the initial response stage. Also, as has been argued by Bago and De Neys (2019), final confidence detection effects may be influenced by the fact that conflict was not only detected but also additional processing might have been applied to rationalize the initial answer and thus attenuating differences in confidence between conflict and no-conflict items. This again might partially explain we did not observe the confidence detection effect in syllogisms at the time of final response. And yet, while we cannot completely eliminate the possibility that the use of the two-response paradigm did somewhat influence the conflict detection results, the findings of our detection efficiency analyses are largely consistent with other studies on individual differences in conflict detection (Frey et al., 2018; Šrol \& De Neys, 2019). Therefore, we believe that the conclusions we present are not invalidated by this element of our research design.

While the proponents of the default-interventionist model did not traditionally make attempts to account for individual differences in intuitive processing (e.g. Evans \& Stanovich, 2013), some of the recently proposed hybrid dual process models can be used to conceptualize this crucial component of reasoning performance. For example, Thompson and Newman (2018) argue that both individual and task specific factors likely influence the relative strength of 
different types of intuitive processing. Patterns of individual differences both in detection efficiency and initial reasoning accuracy which we report here in our view suggest that some reasoners, particularly those with highly instantiated mindware, seem highly efficient in intuitive logical processing (see also Thompson et al., 2018). Considering the task factors, we did not specifically seek to compare the extent of intuitive logical processing on the two types of reasoning problems in our study, but we did notice a remarkable similarity in the results in this regard. Conflict detection data seemed to suggest that participants on a group-level had a slightly harder time to detect conflict in syllogisms in comparison with base-rate neglect tasks with extreme ratios (see also Šrol \& De Neys, 2019). This would be consistent with the notion that extreme base-rates are mostly intuitively processed (e.g. Evans, 2018). And yet, the difference in the amount of intuitive logical processing between the two reasoning problems did not appear when considering initial response accuracies in which, if anything, participants were more accurate in their initial response to syllogisms than base-rate problems. This might be, however, because we used relatively simple syllogisms in our study. In more difficult tasks, such as complex syllogisms or base-rate neglect items with more modest rations, it would be reasonable to expect logical processing to be less available at the intuitive reasoning stage (Thompson \& Newman, 2018; Trippas et al., 2017). When reasoning with complex problems, it is likely that even participants with relatively highly pronounced mindware would not be able to intuitively process the logic of such tasks, thus leading to a higher possibility of detection failure and lower accuracy of initial response even among cognitively disposed participants.

Of course, we should note that our findings regarding the predictors of initial reasoning accuracy cannot be interpreted to reflect pure individual differences in intuitive logical processing. As was correctly remarked by Thompson and Newman (2018), instructions to respond intuitively, restricted time, or even working memory load for that matter, can only limit the possibility for analytic engagement but can by no means be argued to eliminate this type of processing entirely. Then again, some amount of type 2 processing is always implicated when solving reasoning problems, even if only to read the wording of the task and select the response (De Neys, 2018). Experimental manipulations designed to limit the type 2 engagement are also unlikely to completely knock out some shallow analytic processing (i.e. serial associative cognition, Stanovich et al., 2016), as this can presumably take place relatively quickly and with little use of working memory resources. The point in using the two-response paradigm in the present study was that the demanding type 2 operations, such as 
response inhibition and hypothetical thinking, are largely eliminated under a strict deadline. These are precisely those operations which were according to the default-interventionist model thought to be responsible for the link between individual difference predictors and sound reasoning (Evans \& Stanovich, 2013; Stanovich, 2009). Our results, however, show that even in situations when capacity demanding type 2 operations are largely unavailable, reasoners with well-instantiated mindware, better detection efficiency, and high in cognitive reflection are still more likely to provide a normatively correct response in conflict reasoning problems. Still, it would be beneficial in further research to try to replicate our results with the use of several manipulations to limit type 2 processing at once, similarly as was recently done by Bago and De Neys (2017, 2019), to even more rigorously disentangle the individual difference factors which contribute to intuitive and analytic processing.

We are aware of several shortcomings of our study, which we would like to mention at this point. For one, it is likely that the thinking disposition indicators here were unrelated to reasoning accuracy because we used short measures to tap participants' inclination toward analytic and intuitive thought. However, despite the fact that both of our thinking disposition indicators consisted of only five items, they did show good internal consistency. Also, the exact same materials were previously shown to be correlated with reasoning performance, as well as with other conceptually relevant variables (Šrol \& De Neys, 2019). Secondly, our mindware instantiation measure was of very poor reliability. Partly, this is likely because it only consisted of four items on which additionally participants showed very high average performance, thus giving rise to the possible ceiling effect. Nevertheless, it is also undeniably the result of mindware instantiation being substantially task specific (Stanovich, 2018), as low reliability for mindware measure was also reported previously by Šrol and De Neys (2019). And yet, as was the case in their study, mindware instantiation here did show up to be substantially related to detection efficiency and predict reasoning performance over and above other individual difference predictors. This in our view provided reasonable grounds for retaining the mindware instantiation measure in our study despite its low internal consistency.

Also, as mentioned above, we are aware that we did not use a secondary cognitive task to burden participants' working memory. One might suggest that the time restriction and instruction to respond intuitively do not limit the type 2 processing enough and that our participants could still benefit from it at the time of their initial responses to reasoning tasks. This argument was already challenged by Bago \& De Neys (2017). The authors used multiple variations of experimental manipulations across four experiments and their results showed 
that the time restriction itself was as effective for knocking out type 2 processing as either cognitive load, or even as a combination of time restriction and cognitive load manipulations. Moreover, we should mention that the proportions of various direction of change categories in our study were very similar to those observed by Bago and De Neys (2017) under conditions of combined time restriction and cognitive load. This, in our view, provides further evidence that our time restriction manipulation was successful at knocking out type 2 processing.

Since early works by Stanovich and West (2000), studies on individual differences in susceptibility to cognitive biases have assumed an integral role in the research on reasoning and decision making and contributed to the formulation of dominant models of such higher cognitive processes. Under the prominent default-interventionist model, cognitive factors were thought to be predominately related to reasoning performance through their role in type 2 processing (e.g. Evans \& Stanovich, 2013). More recent evidence, however, suggests that substantial individual differences between reasoners exist already at early stages of the reasoning process, at the time when demanding analytic operations could presumably not yet have taken place. The present study contributes to this line of research by identifying mindware instantiation, cognitive reflection, and detection efficiency as predictors of such intuitive logical processing. Our results align with recent notions which implicate mindware instantiation not only as a key predictor of reasoning performance itself but also as an important determinant of the effectiveness of other reasoning components, such as the strength of the intuitively cued logical response and conflict detection ability (Stanovich, 2018; Stanovich et al., 2016). More generally, the findings support recent hybrid dual-process models in which individual difference factors are thought to determine the relative strength of heuristically and logically cued responses already at early intuitive stages of the reasoning process (De Neys, 2018; Pennycook, 2018; Thompson \& Newman, 2018).

\section{REFERENCES}

Bago, B., \& De Neys, W. (2017). Fast logic ?: Examining the time course assumption of dual process theory. Cognition, 158, 90-109.

Bago, B., \& De Neys, W. (2019). Advancing the specification of dual process models of higher cognition: a critical test of the hybrid model view. Thinking \& Reasoning. https://doi.org/10.1080/13546783.2018.1552194

Bago, B., Frey, D., Vidal, J., Houdé, O., Borst, G., \& De Neys, W. (2018). Fast and slow 
thinking: Electrophysiological evidence for early conflict sensitivity. Neuropsychologia, 117, 483-490.

Chandler, J., Mueller, P., \& Paolacci, G. (2014). Nonnaïveté among Amazon Mechanical Turk workers: Consequences and solutions for behavioral researchers. Behavioral Research Methods, 46(1), 112-130.

De Neys, W. (2012). Bias and Conflict: A Case for Logical Intuitions. Perspectives on Psychological Science, 7(1), 28-38.

De Neys, W. (2018). Bias, conflict, and fast logic: towards a hybrid dual process future? In W. De Neys (Ed.), Dual Process Theory 2.0 (pp. 47-65). New York: Routledge.

De Neys, W., Moyens, E., \& Vansteenwegen, D. (2010). Feeling we're biased: autonomic arousal and reasoning conflict. Cognitive, Affective \& Behavioral Neuroscience, 10(2), $208-216$.

Epstein, S., Pacini, R., Denes-Raj, V., \& Heier, H. (1996). Individual differences in intuitiveexperiential and analytical-rational thinking styles. Journal of Personality and Social Psychology, 71(2), 390-405.

Evans, J. S. B. T. (2012). Dual-process theories of deductive reasoning: Facts and fallacies. In K. J. Holyoak \& R. G. Morrison (Eds.), The Oxford Handbook of Thinking and Reasoning (pp. 115-133). Oxford: Oxford University Press.

Evans, J. S. B. T. (2018). Dual process theory: perspectives and problems. In W. De Neys (Ed.), Dual Process Theory 2.0 (pp. 137-155). New York: Routledge.

Evans, J. S. B. T., \& Stanovich, K. E. (2013). Dual-Process Theories of Higher Cognition: Advancing the Debate. Perspectives on Psychological Science, 8(3), 223-241.

Faul, F., Erdfelder, E., Lang, A.-G., \& Buchner, A. (2007). G* Power 3 : A flexible statistical power analysis program for the social, behavioral, and biomedical sciences. Behavioral Research Methods, 39(2), 175-191.

Franssens, S., \& De Neys, W. (2009). The effortless nature of conflict detection during thinking. Thinking \& Reasoning, 15(2), 105-128.

Frederick, S. (2005). Cognitive Reflection and Decision Making. Journal of Economic Perspectives, 19(4), 25-42.

Frey, D., \& De Neys, W. (2017). Is Conflict Detection in Reasoning Domain General ? In Proceedings of the Annual Meeting of the Cognitive Science Society, 39 (pp. 391-396).

Frey, D., Johnson, E. D., \& De Neys, W. (2018). Individual differences in conflict detection during reasoning. Quarterly Journal of Experimental Psychology, 71(5), 1188-1208.

Handley, S. J., Newstead, S. E., \& Trippas, D. (2011). Logic, beliefs, and instruction: a test of the default interventionist account of belief bias. Journal of Experimental Psychology: 
Learning, Memory, and Cognition, 37(1), 28-43.

Johnson, E. D., Tubau, E., \& De Neys, W. (2016). The Doubting System 1: Evidence for automatic substitution sensitivity. Acta Psychologica, 164, 56-64.

Kahneman, D. (2011). Thinking, fast and slow. New York: Penguin Books.

Klaczynski, P. A. (2014). Heuristics and biases: Interactions among numeracy, ability, and reflectiveness predict normative responding. Frontiers in Psychology, 5, 1-13.

Klose, J., Černochová, D., \& Král, P. (2002). Videňský maticový test. Praha: Testcentrum.

Mevel, K., Poirel, N., Rossi, S., Cassotti, M., Simon, G., Houdé, O., \& De Neys, W. (2014). Bias detection: Response confidence evidence for conflict sensitivity in the ratio bias task. Journal of Cognitive Psychology, 27(2), 227-237.

Newman, I. R., Gibb, M., \& Thompson, V. A. (2017). Rule-Based Reasoning Is Fast and Belief-Based Reasoning Can Be Slow: Challenging Current Explanations of Belief-Bias and Base-Rate Neglect. Journal of Experimental Psychology: Learning, Memory, and Cognition, 43(7), 1154-1170.

Pennycook, G. (2018). A perspective on the theoretical foundation of dual process models. In W. De Neys (Ed.), Dual Process Theory 2.0 (pp. 5-27). New York: Routledge.

Pennycook, G., Cheyne, J. A., Barr, N., Koehler, D. J., \& Fugelsang, J. A. (2014). Cognitive style and religiosity: the role of conflict detection. Memory \& Cognition, 42(1), 1-10.

Pennycook, G., Fugelsang, J. A., \& Koehler, D. J. (2012). Are we good at detecting conflict during reasoning? Cognition, 124(1), 101-106.

Pennycook, G., Fugelsang, J. A., \& Koehler, D. J. (2015). What makes us think? A threestage dual-process model of analytic engagement. Cognitive Psychology, 80, 34-72.

Sinayev, A., \& Peters, E. (2015). Cognitive reflection vs. calculation in decision making. Frontiers in Psychology, 6, 532.

Šrol, J. (2019). Individual differences in susceptibility to cognitive biases: Implications for theories of rational thought. Unpublished doctoral thesis. Bratislava: Comenius University.

Šrol, J., \& De Neys, W. (2019). Predicting individual differences in conflict detection and bias susceptibility during reasoning. https://doi.org/10.31234/osf.io/2uf6g

Stanovich, K. E. (2009). Distinguishing the reflective, algorithmic, and autonomous minds: Is it time for a tri-process theory? In J. S. B. T. Evans \& K. Frankish (Eds.), In two minds: Dual processes and beyond (pp. 55-88). Oxford University Press.

Stanovich, K. E. (2018). Miserliness in human cognition: The interaction of detection, override and mindware. Thinking and Reasoning, 24(4), 423-444. 
Stanovich, K. E., \& West, R. F. (2000). Individual differences in reasoning: Implications for the rationality debate? Behavioral and Brain Sciences, 23(5), 645-665.

Stanovich, K. E., West, R. F., \& Toplak, M. E. (2011). Individual differences as essential components of heuristics and biases research. In K. Manktelow, D. E. Over, \& S. Elqayam (Eds.), The Science of Reason: A Festschrift for Jonathan St. B. T. Evans. (pp. 355-396). Psychology Press.

Stanovich, K. E., West, R. F., \& Toplak, M. E. (2016). The Rationality Quotient: Toward a test of rational thinking. MIT Press.

Thompson, V. A., \& Johnson, S. C. (2014). Conflict, metacognition, and analytic thinking. Thinking \& Reasoning, 20(2), 215-244.

Thompson, V. A., \& Newman, I. R. (2018). Logical intuitions and other conundra for dual process theories. In W. De Neys (Ed.), Dual Process Theory 2.0 (pp. 121-136). New York: Routledge.

Thompson, V. A., Pennycook, G., Trippas, D., \& Evans, J. S. B. T. (2018). Do smart people have better intuitions? Journal of Experimental Psychology: General, 147(7), 945-961.

Thompson, V. A., Prowse Turner, J. A., \& Pennycook, G. (2011). Intuition, reason, and metacognition. Cognitive Psychology, 63(3), 107-140.

Toplak, M. E., West, R. F., \& Stanovich, K. E. (2011). The Cognitive Reflection Test as a predictor of performance on heuristics-and-biases tasks. Memory \& Cognition, 39(7), $1275-1289$.

Trippas, D., \& Handley, S. J. (2018). The parallel processing model of belief bias: review and extensions. In W. De Neys (Ed.), Dual Process Theory 2.0 (pp. 28-46). New York: Routledge.

Trippas, D., Thompson, V. A., \& Handley, S. J. (2017). When fast logic meets slow belief : Evidence for a parallel-processing model of belief bias. Memory \& Cognition, 45(4), 539-552.

Tversky, A., \& Kahneman, D. (1974). Judgment under Uncertainty: Heuristics and Biases. Science, 185, 1124-1131. 